ciated with subsequent non-adherence with the treatment regime. Further, such an approach may prove divisive for the clinical team, with members having different views about its advisability. Such uncertainty and ambivalence among team members is likely to have an adverse effect on the patient. In the longer term, the most positive outcome is that the patient shows persistent clinical improvement, and willingly adheres with both medication and the blood tests, as the reasons for the original reluctance to take clozapine no longer hold sway. In theory, there are several plausible explanations for such a change. For example, if the objections to clozapine or the blood tests were delusionally-based they might be overcome by a reduction in the influence and intensity of the delusions as part of the treatment response. If the original concern was about possible sideeffects, the patient may be reassured by the experience of taking the medication. However, in the absence of any published data on the clinical outcome following the initial enforcement of a clozapine regime, such long-term benefits remain theoretical. The potential for a positive outcome needs to be balanced against the possible long-term adverse consequences of enforcing clozapine. The drug may fail to achieve the degree of improvement (in terms of reduction in the intensity of relevant delusional ideas, improvement in insight, etc.) that would render the patient likely to comply with medication over time. If therapeutic relationships with the multidisciplinary team are not re-established, the patient may disengage from treatment and any broader rehabilitation programme. Even if this does not occur, discussions between members of the clinical team and the patient may become limited to an unproductive debate about adherence to the medication regime, at the expense of consideration of wider issues related to rehabilitation and social integration.

While the potential risks and benefits of enforcing clozapine are uncertain in those patients who have never been exposed to the drug, more reliable predictions may be made for those who have already received an adequate trial. For patients previously showing an impressive improvement with clozapine, who are otherwise unresponsive to antipsychotic medication, it could be argued that the justification is rather greater (Barnes et al, 1996). This may be particularly so for patients who pose a 'substantial risk of harm to others or themselves' related to the severity of their psychotic illness. In such cases, the successful use of electroconvulsive therapy to gain a temporary improvement in the psychotic illness, allowing cooperation with the clozapine regime, has been reported (Green et al, 1994; James \& Gray, 1999).

\section{References}

BARNES, T. R. E., MCEvedy, C. J. B. \& NELSON, H. E. (1996) Management of treatment resistant schizophrenia unresponsive to clozapine. British Journal of Psychiatry, 169 (suppl. 31), 31-40.

Green. A. I., Zalma, A., Berman, I., et al (1994) Clozapine following ECT: A two-step treatment. Journal of Clinical Psychiatry. 65, 388-390.

JAMES, D. V. \& GRAY, N. S. (1999) Elective combined electroconvulsive and clozapine therapy. International Clinical Psychopharmacology. 14, 69-72.

Thomas R. E. Barnes, Professor of Clinical Psychiatry. Department of Psychiatry. Division of Neuroscience and Psychological Medicine, Imperial College School of Medicine, Academic Centre, Ealing Hospital, St Bernard's Wing. Uxbridge Road, Ealing UB1 3EU

\title{
Early detection of antipsychotic side-effects
}

\author{
Robert Chaplin, Julie Gordon and Tom Burns
}

\begin{abstract}
Aims and methods Staff from five community mental health teams (CMHTs) were trained to use structured rating scales for akathisia, tardive dyskinesia and Parkinsonism. Detection rates of these side-effects were compared for the six months before and after the intervention.
\end{abstract}

Results Fifty-seven per cent of the target professionals participated, screening 200 (52\%) elligible patients. This resulted in significant increases in the recording of all three side-effects as positive but no increase in their formal diagnosis. 
Clinical implications Detection rates of these sideeffects can be increased to those predicted by research with significant reductions in drug dose and non-adherence and without clinical deterioration.

Toxic effects of antipsychotic drugs are frequently missed (Weiden et al, 1987) leading to suffering of patients, litigation (Dillon, 1992) and poor adherence with treatment (Fleischhacker et al, 1994). Detection rates of akathisia, tardive dyskinesia and Parkinsonism have been improved by training medical staff (Dixon et al, 1989) while nurses (Dillon, 1992) can be successfully trained to screen for tardive dyskinesia. We hypothesised that all mental health staff could be trained to screen for tardive dyskinesia, akathisia and Parkinsonism. We evaluated the outcome on the side-effect detection rate, prescribing behaviour and clinical stability.

\section{The study}

A single examination was proposed to rate both Parkinsonism on a shortened Simpson and Angus Scale (Simpson \& Angus, 1970) and tardive dyskinesia on the Abnormal Involuntary Movement Scale (AIMS; Guy, 1976). The Barnes Akathisia Scale (Barnes, 1989) was chosen for its popularity, speed of administration and reliability. An annual examination was adopted as suggested by Lader (1994). Non-medical staff were offered training in the use of these scales on the recommendation of the Clinical Standards Advisory Group's (CSAG) report on the treatment of schizophrenia (CSAG, 1995).

After a brief presentation to each community mental health team (CMHT), teaching sessions with the whole teams were arranged, lasting 60-90 minutes. This involved education about antipsychotic medication, rational prescribing, side-effects and their complications. A video showing side-effects was rated and discussed in the group. Written instructions were provided on how to perform and rate each examination and interpret the scores. Keyworkers were advised to discuss positive results with a psychiatrist. Individual teaching on-site was offered to all participants.

Probable tardive dyskinesia was defined by mild abnormal movements in two body areas or moderate abnormal movements in one body area on the AIMS. A rating of at least two (mild) on the global item of the Barnes Akathisia Scale was taken as evidence of akathisia. The Simpson and Angus scale was restricted to items of wrist rigidity, elbow rigidity, gait, salivation and tremor. A further item, cog-wheeling, was added and rated as either zero (absent) or one (present). Parkinsonism was defined by the presence of cog-wheeling or a rating at least two on one of the other five items. This required a slightly higher mean score $(0.4)$ for the diagnosis of Parkinsonism than the original rating by Simpson and Angus (0.3).

Six CMHTs in the London borough of Wandsworth were targeted. Patients were eligible if they received antipsychotic drugs, regardless of diagnosis or duration of treatment and were identified by their keyworkers. A data recording sheet was entered into their case notes to prompt the keyworker at each appointment. Patients were informed of the study through the patients' council and a local service users magazine. The project ran for approximately six months starting from the days the teams received their first training (July-September 1997). The case notes of those patients who had been screened were then examined for ratings of side-effects on the data sheet and their formal diagnosis. The six months before the training (control period) was compared with the six months immediately afterwards (intervention period) for differences in side-effect detection rates, clinical and prescribing outcomes. Ethics committee approval was obtained and data were analysed using SPSS (Norusis, 1986).

\section{Findings}

Four of original six (and one extra) CMHTs participated. We recruited $31(57 \%)$ of the 54 keyworkers from the five participating teams (see Fig. 1). Social workers were directed by their management not to participate, reducing to a possible 42 keyworkers of whom the 31 recruited represent $77 \%$. Two hundred $(52 \%)$ screening examinations were performed from a total of 383 eligible patients. Community psychiatric nurses screened $160(80 \%)$, doctors $30(15 \%)$ and occupational therapists $10(5 \%)$.

Patients had a mean age of 45 years (s.d. $=13$ years) and were first ill at a mean age of 28 years (s.d.=9 years), 90\% had been treated for at least five years and $70 \%$ for at least 10 years. $57 \%$ were male. By case notes diagnosis, $73 \%$ suffered from schizophrenia, $11 \%$ bipolar disorder and $12 \%$ 'other psychotic disorders'. The mean length of the intervention period was 178 days and the control period 180 days. At the beginning of the intervention period, $18 \%$ of the patients were prescribed atypical antipsychotic drugs exclusively (clozapine, risperidone or olanzapine), with a further $8 \%$ changing to them during the period.

The total number of positive cases of akathisia recorded increased significantly from $13(7 \%)$ in the control to $30(15 \%)$ in the study period $\left(\chi^{2}=8.83, P=0.003\right.$, d.f. $\left.=1\right)$. The recording of a formal diagnosis of akathisia increased (not significantly) to $10 \%$ of the group. The total 


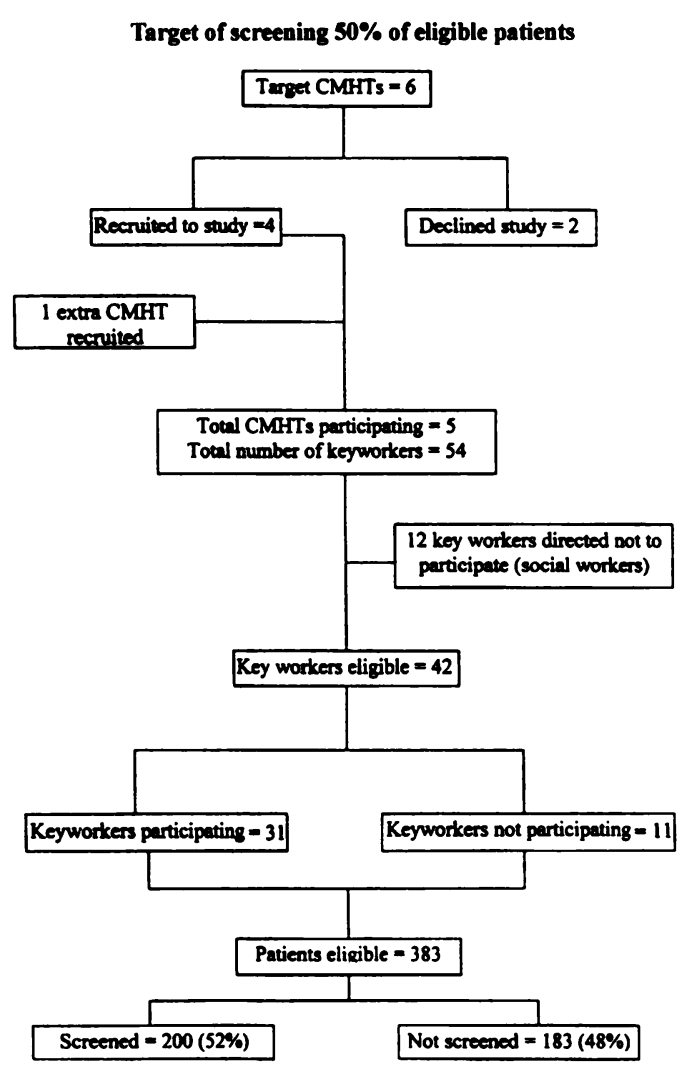

Fig. 1. Flow diagram of recruitment of community mental health teams (CMHTs) and keyworkers and completion of screening targets.

number of posittve cases of Parkinsonism increased significantly from $32(16 \%)$ to $56(28 \%)$ $\left(x^{2}=11.5, P=0.0007\right.$, d.f. $\left.=1\right)$, but without an increase in the recording of a formal diagnosis in the study period (15\%).

At the start of the control period, tardive dyskinesia had been diagnosed in only seven (4\%) of the 200 patients. The incidence of recording new cases of tardive dyskinesia rose from 6 out of $193(3 \%)$ during the control period to 29 out of $187(16 \%)$ in the intervention period (difference between two proportions, $P_{1}=6 / 193$, $P_{2}=29 / 187$, standard error of $P_{1}-P_{2}=0.0318$, $95 \%$ confidence intervals -0.15 to -0.3 , $P=0.0045)$. However, only $10(5 \%)$ patients with new recordings in the intervention period were given the diagnosis of tardive dyskinesia. At the end of the intervention period, $42(21 \%)$ of patients were either diagnosed with tardive dyskinesia or had a positive AIMS result. Overall, we found that $84(42 \%)$ of patients had a positive screening for at least one of the measured side-effects. There was no association between age or gender and posittve screenings of any of the three side-effects.

A further analysis showed no significant differences between atypical and standard antipsychotics in the positive ratings of all three side-effects. Tardive dyskinesia was rated positive for $10(24 \%)$ of patients on atypical and $18(12 \%)$ on standard antipsychotics $\left(\chi^{2}=0.46, P=0.5\right)$. Akathisia was rated positive on four $(10 \%)$ of patients on atypical and $18(12 \%)$ on standard antipsychotics (Fisher's exact test, $P=0.49$ ). Parkinsonism was rated positive on nine $(21 \%)$ of the patients prescribed atypical and $39(25 \%)$ of those on standard antipsychotics $\left(x^{2}=0.23, P=0.6\right)$.

We were concerned that the screening programme might produce adverse clinical outcomes by interfering with the keyworker-patient relationship. We compared four indicators across control and intervention periods in the case-notes: (a) non-adherence for at least two weeks; (b) a clear clinical deterioration; (c) number of non-routine visits; and (d) number of in-patient days. There were no significant differences between any of these indicators except non-adherence which was lower in the intervention $(8 \%)$ than control (13\%) period (McNemar's test, $P=0.035$, d.f. $=1)$. A successful screening programme might also be expected to change the behaviour of clinicians. The average antipsychotic dose change in the control period was an increase of dose of $29 \mathrm{mg}$ chlorpromazine equivalents $(Z=-1.2, P=0.2)$, while in the intervention period the average dose fell significantly by $25 \mathrm{mg}$ $(Z=-2.44, P=0.015)$. There was no significant difference in the number of patients with a change of drugs between the control (24\%) and study $(20 \%)$ periods.

\section{Comment}

Our screening programme has increased the side-effect detection rates to approach those reported in the literature. The prevalence of probable tardive dyskinesia in our study was $21 \%$, similar to the published estimate of $20 \%$ (American Psychiatric Association, 1992). Akathisia has a prevalence of $20-25 \%$ (Halstead et al, 1994) and our posittve screening rate of $15 \%$ may reflect increasing use of atypical antipsychotics. An authoritative figure for the prevalence of Parkinsonism is 20-40\% (Lishman. 1998) and our positive screening rate of $28 \%$ is within this range.

Although recruitment was eventually successful to the study, a number of problems were identified. Some staff expressed concern about touching patients, while others feared litigation arising from their identification of medical conditions they did not feel competent to manage. Less surprisingly, the project was seen as increasing an already busy workload. This 
Table 1. Rates of screening, prescribing and clinical outcomes in control and study perlods

\begin{tabular}{|c|c|c|c|}
\hline & Control period & Intervention period & Signiliconce \\
\hline Duration (days) & 180 & 178 & NS \\
\hline \multicolumn{4}{|l|}{ Side-effects screening } \\
\hline New tardive dyskinesia cases detected & $6(3 \%)$ & 20 (10\%) & $P=0.0045$ \\
\hline $\begin{array}{l}\text { New tardive dyskinesia cases formally } \\
\text { diagnosed }\end{array}$ & $6(3 \%)$ & $10(5 \%)$ & NS \\
\hline Akathisia screening positive & $13(7 \%)$ & 30 (15\%) & $P=0.003$ \\
\hline Akathisia formally diagnosed & $13(7 \%)$ & 20 (10\%) & \\
\hline Parkinsonism screening positive & $32(16 \%)$ & $56(28 \%)$ & $P=0.0007$ \\
\hline Parkinsonism formally diagnosed & $32(16 \%)$ & $29(15 \%)$ & NS \\
\hline \multicolumn{4}{|l|}{ Prescribing outcome } \\
\hline Change of antispychotic drug $\mathrm{Y} / \mathrm{N}$ & 48 (24\%) & $40(20 \%)$ & NS \\
\hline Change of antipsychotic dose $\mathrm{V} / \mathrm{N}$ & $83(42 \%)$ & $72(36 \%)$ & NS \\
\hline Initial antipsychotic dose (mg CPZ) & $506 \mathrm{mg}$ & $535 \mathrm{mg}$ & \\
\hline Final antipsychotic dose (mg CPZ) & $535 \mathrm{mg}$ & $510 \mathrm{mg}$ & \\
\hline Change in antipsychotic dose & $+29 \mathrm{mg}$ & $-25 m g$ & $P=0.015$ \\
\hline \multicolumn{4}{|l|}{ Clinical outcome } \\
\hline Days in hospital & 5.7 & 5.5 & NS \\
\hline Documented clinical deterioration & $47(24 \%)$ & $39(20 \%)$ & NS \\
\hline Number recelving an emergency visit & $22(11 \%)$ & 25 (13\%) & NS \\
\hline Number non-adherent & $26(13 \%)$ & $15(8 \%)$ & $P=0.035$ \\
\hline
\end{tabular}

Wilcoxon and categorical by $\chi^{2}$. CPZ, chlorpromazine equivalents; NS, not significant.

resistance to participation was minimised by frequent contact with staff and on-site training sessions which demonstrated that the assessments were brief and simple to administer.

The failure to use positive screening results to make a formal diagnosis of movement disorders remains the major limitation. It is possible that nurses may not have fully understood the implications of the screening and did not communicate the results to psychiatrists. This study is also limited because the accuracy of ratings by keyworkers was not verified by an independent research assessment. We judged that this level of interference with routine clinical practice would not be acceptable to keyworkers with heavy demands on their time. However, with our prevalence rates similar to those found in the literature, we have concluded that there are no major discrepancies in rating and that a brief training increases the ability to assess sideeffects accurately. Further studies are required to determine appropriate strategies for effective management of antipsychotic side-effects in routine clinical practice.

\section{References}

AMERICAN PSYchIATRIC Association (1992) Tardive Dyskinesia: A Task Force Report of the American Psychiatric Association. Washington, DC: APA.

BARNES, T. R. E. (1989) A rating scale for drug-induced akathisia. British Journal of Psychiatry, 184, 672-676.

Clinical Standards AdVISORY Group (1995) Report of a Clinical Standards Advisory Committee on Schizophrenia. Volumes 1 \& 2. London: HMSO.

Drwon. N. B. (1992) A screening system for tardive dyskinesia: development and implementation. Journal of Psychosocial Nursing and Mental Health Services. $\mathbf{s 0 .}$ 3-7.
Dixon, L., Weiden, P. J., Frances, A. J., et al (1989) Management of neuroleptic induced movement disorders: effects of physician training. American Journal of Psychiatry. 146, 104-106.

FLEISCHHACKER, W. W., MEISE, U., GUNTHER, V., et al (1994) Compliance with antipsychotic drug treatment: influence of side effects. Acta Psychiatrica Scandinavica, 89 (suppl. 382), 11-15.

GuY, W. (1976) ECDEU. Assessment Manual for Psychopathology. Washington, DC: US Department of Health and Welfare.

Halstead, S. M. Barnes, T. R. E. \& Speuer, J. C. (1994) Akathisia: prevalence and associated dysphorla in an in-patient population with chronic schizophrenia. British Journal of Psychiatry, 164, 177-183.

LADER, M. H. (1994) Lundbeck Guidelines for the Management of Schizophrenia. London: Lundbeck.

ushiman, W. A. (1998) Organic Psychiatry: The Psychological Consequences of Cerebral Disorder (3rd edn). Oxford: Blackwell Science.

NORUSIS, M. J. (1986) SPSS/PC+ Statistics. Chicago. IL: SPSS.

Simpson, G. M. \& ANGUS, J. W. S. (1970) A rating scale for extrapyramidal side-effects. Acta Psychiatrica Scandinavica Supplementum, 212, 11-19.

WEIDEN, P. J., MANN, J. J., HAAS, G., et al (1987) Clinical non-recognition of neuroleptic-induced movement disorders: a cautionary study. American Joumal of Psychiatry, 144, 1148-1153.

*Robert Chaplin, Consultant Psychiatrist, Julie Gordon, Research Nurse, South West London \& St George's Mental Health Service, Springfield Hospital, 61 Glenburnie Road, London SW17 7DJ; and Tom Burns, Professor of Community Psychiatry. Department of Psychiatry. St George's Hospital, London

\section{"Correspondence}

